\title{
ANALYSIS OF DATA QUALITY ON ADVERSE DRUG REACTIONS AND OF METHODS FOR ITS IMPROVEMENT
}

\author{
Mark Tkachenko, Natalia Dobrenko, Daria Butorina, Elvira Bulatova, \\ Aleksandra Vatian and Anton Arazashvili \\ ITMO University, Sankt-Petersburg 197101, Russia
}

\begin{abstract}
This article presents the results of quality analysis of data on adverse drug reactions (ADRs) for groups of drugs, which are most frequently discussed by users of Internet resources.

Several Russian-language resources were used as data sources. An open database MedDRA, translated into Russian, was used as a database of ADRs. To collect information about ADRs from Internet resources, a rule-based algorithm has been developed and implemented. Venn diagrams, multigraphs of pairwise as well as interaction scaling analysis were used to compare data on ADRs obtained from different sources. To assess the quality of the data, conditionally objective indicators were used, such as relevance, integrity, compatibility, measurability, and source characteristic.

As a result, significant violations of the integrity and measurability of data were identified, and a comparative assessment of the characteristics of data sources was carried out. The ways of improving the quality of data on ADRs have been formulated.
\end{abstract}

\section{KEYWORDS}

Adverse Drug Reactions, Pharmacovigilance, Data Quality

\section{INTRODUCTION}

Adverse drug reactions (ADR) are a significant source of morbidity and mortality, and their constant monitoring and accounting in real medical practice is required in accordance with the recommendations of the World Health Organization (WHO).

Information on ADRs used to be collected by health professionals during clinical trials of drugs. However, research and clinical practice in recent years expanded the list of ADR sources and the time limit for accepting reports. Pharmacovigilance now must not only record ADR data provided by doctors, but also collect patient opinions. As data sources, not only official protocols can now be used, but also social networks, forums, blogs and other means of Internet communication.

ADRs need to be monitored after official approval, especially due to new drugs appearing on the market and the spreading threat of self-medication. Such heterogeneity and diversity of collected data can objectively reduce its quality, which is not always taken into account to a sufficient degree when processing this data and using it for practical applications.

The quality of medical data in general [Romero, Na Hong, Van den Berghe, Mavrogiorgou] and ADR data in particular [Soyer, Jeong, Oh] is a subject or worldwide research. However, it is only beginning to gain traction in Russia [Antipov]. This article presents the results of quality analysis of data on adverse drug reactions (ADRs) for two groups of drugs: lipid-lowering drugs, antiarrhythmic drugs, which are most frequently discussed by users of Internet resources. 


\section{BACKGROUND AND RELATED WORKS}

\subsection{Means for Collecting ADR Information}

Data collection systems for ADRs vary widely across countries. One of the most advanced countries in this regard is the United States of America. Russia was chosen for comparison for the following reasons. In the USSR, there was a system of state control of the quality of medicines, but with the collapse of the USSR it was almost entirely destroyed and in Russia is at a new stage of development [Lepakhin]. In addition, Russian legislation on the sale of medicine can be considered rather liberal, and many drugs that are available in other countries only with a prescription are sold in Russia without one. This allows for self-treatment and treatment based on data found on the Internet, where patients self-diagnose themselves and choose which drugs to take based on the opinion of other social network users.

The results of the analysis of available information sources on ADR and means of their processing in English and in Russian are briefly described in detail below. More complete information can be found in [Vatian].

There are several channels for collecting information about ADRs, which can be divided into two categories: information from official sources and information from individuals. In 2016, Russia entered the Good Pharmacovigilance Practice rules [GVP]. According to its norms, the participants of the circulation of medicines should report to the specialized center [Roszdravnadzor] about all cases of adverse effects not listed in the drug leaflet, serious and unexpected adverse reactions, special interactions with other drugs encountered in clinical trials and during clinical use of drugs. However, this system is aimed not so much at fixing individual cases of ADRa as on withdrawing poor-quality medicine batches from sale. The individual doctors' and patients' experience is not included in this database. In this regard, the role of practitioners and patients as information providers for the pharmacovigilance system increases sharply [Foster].

People express their opinion about medications, including ADR, primarily in the form of texts. It can be specialized medical texts, including doctors' conclusions and health records, as well as entries in social media like forums, blogs, etc. Natural lan-guage processing (NLP) methods are used worldwide due to their effective automated processing. NLP methods have seen success when used for the task of processing medical texts, English texts regarding ADRs in particular. At the same time, with respect to other languages, the progress is slower, which has negative consequences in the context of globalization and population migration growth.

Steps are being taken to improve this situation, primarily to unify worldwide approaches to ADRs. For example, American Medical Informatics Association launched a special didactic panel called "Clinical Natural Language Processing in Languages Other Than English" [AMIA]. In the manifesto of this panel, Germanic, Romance, Sino-Tibetan and Afro-Asiatic languages are mentioned, but not Russian.

In the Russian-language segment, studies on the use of NLP in medicine are underway, but only a few are focused on ADR detection. For example, in [Baranov], clinical records are analyzed in order to solve problems such as indicating the absence or the presence of disease in the patient and the severity of the course of the disease. The work [Lushnov] is aimed at monitoring the patient's state of health in different contexts: the treatment and diagnostic process, clinical trials of the new treatment regimen, monitoring the work of the clinic by its management.

\subsection{Means for Evaluating ADR Degree}

Problems associated with evaluating the degree of ADRs are widely discussed in various works [32-35]. The main problem researchers face is determining the causal relationship between drugs and undesirable clinical events, and in this regard, clinical trials are ongoing [36, 37].

There are official guidelines for evaluating drug-drug interactions (DDI) at the drug development level. DDI can result in alterations of either drug pharmacokinetics (PK), pharmacodynamics (PD) or both, and evaluations can be done in vitro and in vivo studies [Talbot, Marante, Khan, Prueksaritanont]. However, at the stage of using drugs in clinical practice, the main means of estimation is expert judgment [Khan]. Methods are being developed to improve the quality of group examination be increasing the intra rater agreement of a group of experts, but the opinion of each individual expert is evaluated in a binary scale [Khan]. 
Based on expert opinions or the results of the analysis of the chemical structure of the active substances, matrices of pairwise interactions between drugs are constructed, which allow algorithmically estimating ADR levels. For example, in the article [Zhang], several matrices of interactions between medicinal substances are built on the principle of "each with each". The authors used only official sources of information: the FAERS database and the PubChem chemical structure database. Thus, each drug was represented by a binary adverse effect profile (4,192-dimensional for labeled effect, 10,093-dimensional for off-label effect, 881 for chemical structures). The authors proposed label propagation algorithms addressing the following problem: given an undirected weighted network with $n$ nodes where a small portion of them are labeled, estimate the labels of the rest of the unlabeled nodes. Thus, it becomes possible to evaluate the toxicity of any pair of unlabeled drugs. However, to achieve this, the parameters that determine the influence of a node's neighbors relative to its provided label need to be known, as well as the importance of each drug similarity matrix.

Based on this and similar methodologies, software has been developed and applied to evaluate DDIs, such as [Micromedex]. However, all of these tools are proprietary, are based on US medical standards and are not localized for non-English speaking countries, which makes them less accessible for medical professionals in Russia.

Various scales are proposed for assessing the danger level of ADR manifestation. For example, [Micromedex] uses two scales: a four-level scale of severity levels and a three-level scale of documentation levels (scientific evidence), by which the scientific validity of the claimed ADR degree is understood. A wider range of scales is offered by WHO [Marante]. WHO introduces four general categories of causality: Related, Probable (Likely), Possible, Unlikely, Unrelated. In addition, according to WHO, there are two more types of causality categories, which are not applicable for clinical research: Conditional/Unclassified (if more data is needed for proper assessment or when additional data is being analyzed) and Non-accessible/Unclassifiable (if the information is insufficient or contradictory and cannot be completed or verified). WHO argues that causality can be classified only as "Related" or "Not Related" for clinical research in some countries and spontaneous reports.

Finally, the initial information for scaling is context-sensitive [Marante]. All of the above complicates the problem of constructing an ADR rating scale, localized for a particular country and accessible to the end user, whether it is a doctor or a patient.

Thus, the authors of the article set themselves the following tasks:

- conduct a comparative analysis of data on ADR obtained from different sources available in Russia;

- at a substantive level, evaluate the quality of ADR data available in Russia;

- outline ways to improve Russian ADR data quality.

\section{METHODS AND MATERIALS}

The following resources were used as data sources: official sources - Russian Medicines Register [RMS] and manufacturers' instructions for specific medicines, and Internet sources - large Internet forums containing reviews about medicines [Doctors], articles about specific medicines on the Wikipedia.org website, as well as the APIs of VK, Russia's largest social network. We used an open database MedDRA, translated into Russian, as a database of side effects.

To collect information about side effects from Internet resources, a rule-based algorithm has been developed and implemented. The algorithm considers not only the mentions of side effects, but also the presence of words that may deny the side effect. The experimentally estimated accuracy of the constructed parser was $83 \%$.

In order to compare data on ADR obtained from different sources we used (1) Venn diagrams showing the number of matches in the identified ADR from different sources; (2) graphs of pairwise interactions and analysis of their connectivity; (3) scaling interactions analysis. We used two types of graphs. The first type was based on the entities "medicine - side effects" and shows the presence of a connection between them. To build the graph, we used the networkx (graph construction) and plotly (graph visualization) Python libraries. An example of a graph is presented in Figure 1. The violet-colored nodes correspond to drugs, and pink-colored nodes correspond to their side effects. 
The second type of graph is based on the entities "drug - drug", and the link between a pair of vertices reflects the presence of contraindications to their joint use. The graph is based on a complete database of drug interactions, whose full structure is not given in the article due to cumbersome. The database is built on data taken from the Russian Medicines Register site and contains 2187 drug interactions. To build the graph, the tables drug_substance, drug_substance2, type and the python pandas library were used. The graph in a simplified form is shown in Figure 2. Based on the full graph, one can construct partial subgraphs, which related to a specific drug or a specific degree of interaction (Figure 3, a, b). The edges between the vertices were colored to mark the degree of mutual influence of drugs.

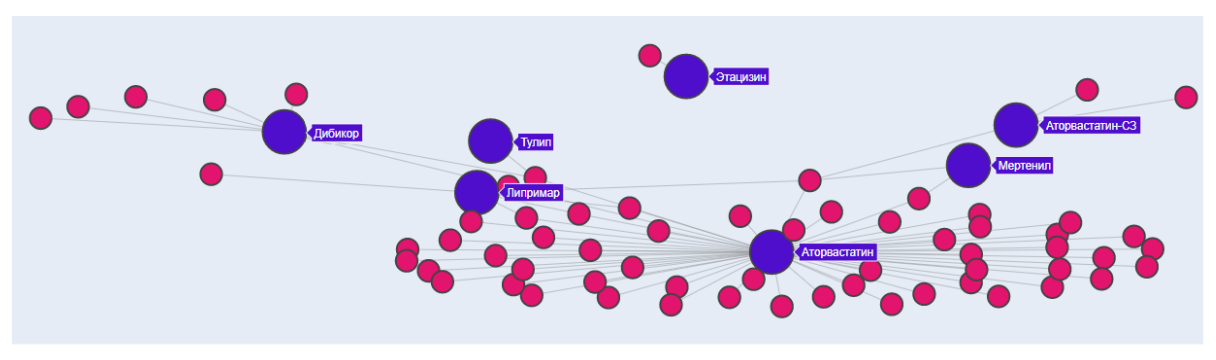

- Drugs $\bigcirc$ side_effect

Figure 1. An example of a "drug - side effects" relationship graph

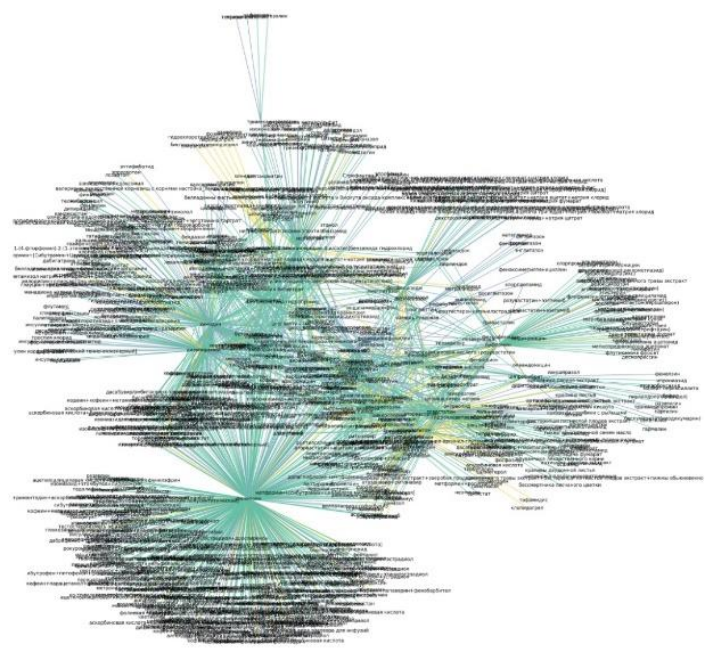

Figure 2. Graph of interactions of medicial substantions under consideration
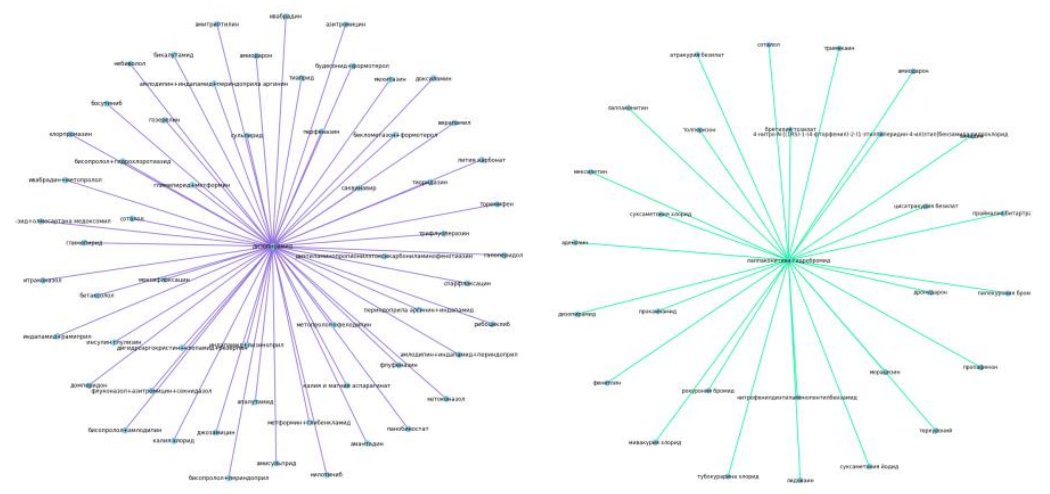

Figure 3. Examples of graphs of interactions of medicinal substances: a- disopyramids, major interactions; $\mathrm{b}$ - lappaconitine hydrobromide, moderate interactions 
To assess the quality of the data [Batini, Mosley, Romero, Na Hong, Van den Berghe], we used the following indicators:

- Actuality of data - a designated or indirectly determined point in time in which the data reflects the real state of the target object.

- Relevance of data - how the data on the real state of the target object corresponds to the problem being solved.

Data integrity - the completeness of reflection of the real state of the target object in terms of semantic consistency of data and compliance with a given format.

- Measurability of data - the presence of qualitative or quantitative characteristics in the data of the real state of the target object.

- $\quad$ Source characteristics.

\section{RESULTS AND DISCUSSION}

\subsection{Analysis of ADR Data Integrity}

As shown by the analysis, data completeness is one of the key problems that reduce the quality of ADR data, and it can take place with varying degrees of severity [Soyer]. Note that the problem affects not only spontaneous sources of information about ADRs [Oh], but also professional ones. For example, [Soyer] states discrepancies between the ADRs identified by medical records technicians and the ADRs identified by the pharmacovigilance team.

Data integrity is not fully ensured even in an official source of medical information as the Russian Medicines Register. For example, among the groups of drugs considered in this article, adenosine and lappaconitine hydrobromide are presented, the simultaneous administration of which can cause side effects, however, mention of ADR is contained only in the description of lappaconitine hydrobromide and is not in the description of adenosine. In addition, a number of drugs are presented in the Russian Medicines Register, the interactions of the constituent substances of which are unexplored. It should be noted that the Russian Medicines Register serves as a source for drawing up instructions for medicines that are sold in pharmacies and are easily accessible to end consumers without medical supervision.

To study the integrity of ADR data most accessible to end consumers, we com-pared the data related to Atorvastatin from all the sources mentioned in Section 3. The eliciting of ADR data from the Wikipedia article and from the official instruction for the drug was carried out by experts. To retrieve ADR data from online forums and from the social network VK a rule-based algorithm was implemented. After text preprocessing, the algorithm implements the following rule: a side effect is present if the word denoting it is present in the text and there are no words negating the side effect. The algorithm was preliminarily tested on a Russian-language database compiled by an expert method, with the final F-score of 0.82 . The results are presented in the Table 1. The Algorithm line corresponds to the application of the written algorithm to Internet forums, the Algorithm+VK line corresponds to the application of the algorithm to the VK network.

Table 1. Information on ADR for Atorvastatin obtained from different sources

\begin{tabular}{|c|c|}
\hline Source & Adverse effects \\
\hline Wikipedia & $\begin{array}{l}\text { insomnia, headache, asthenic syndrome; nausea, diarrhea, abdominal pain, dyspepsia, flatulence, } \\
\text { constipation; myalgia }\end{array}$ \\
\hline Instruction & $\begin{array}{l}\text { constipation, flatulence, dyspepsia and abdominal pain. headache, asthenic syndrome, insomnia, } \\
\text { dizziness }\end{array}$ \\
\hline SIDER & $\begin{array}{l}\text { nasopharyngitis, pain in the extremities, urinary tract infection, musculoskeletal pain, muscular } \\
\text { spasm, insomnia, pharyngolaryngeal pain }\end{array}$ \\
\hline Algorithm & necrosis, myocardium, atherosclerosis, pain, surgery \\
\hline Algorithm+VK & $\begin{array}{l}\text { pain, hemiplegia, myocardium, irritability, inflammation, gout, bedridden, arthritis, separation, } \\
\text { atrophy, osteoporosis, stress, disability, drop, aneurysm, hypertension, paralysis, cough, ulcer, } \\
\text { tachyarrhythmia, depression, thyroiditis, hepatitis, hypertonic, atherosclerosis, trauma, alcoholism, } \\
\text { obesity, numbness, dehydration, pancreatitis, toothache, myasthenia gravis, gas, diuresis, acne, } \\
\text { drowsiness, infectious disease, eczema, overdose, psoriasis, constipation, headache, fatigue, suicide, } \\
\text { irritability, death, migraine, lethargy, asthma,, neuropathy, hallucination }\end{array}$ \\
\hline
\end{tabular}


The comparison results are graphically presented in Figure 4 in the form of Venn diagram. The diagram shows the coincidence in the lists of side effects of the same medication (Atorvastatin), mentioned in different sources. To determine the similarity measure of side effects detected from various sources, we used the Jacquard coefficient $K_{i j}=c a+b c$, where $a$ is the number of side effects in group $i, b$ is the number of side effects in group $j, c$ is the number of side effects values common for groups $i$ and $j$. On the Venn diagram, for each two, three, four, and five sources $c$ values are shown.

Table 1 shows that the coincidence in the lists of adverse effects of the same drug, mentioned in different sources, is rather weak. This is also confirmed by the Jacquard coefficients (see Figure 4). It is noteworthy that the list of side effects highlighted by users of the VK network significantly exceeds the lists provided by official sources. This fact can serve, as an indirect confirmation that the information on ADRs received from end consumers, at least, cannot be ignored.

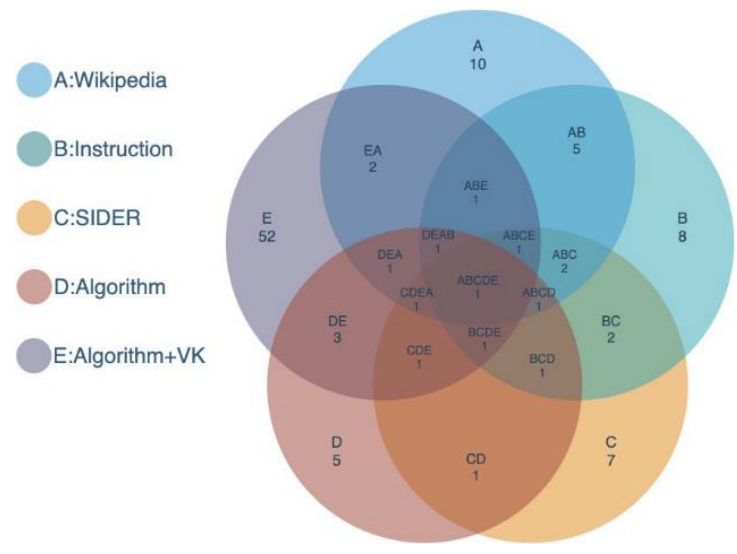

Figure 4. Venn diagram for comparing information on side effects of astrovastatin obtained from various sources

\subsection{Analysis of ADR Data Measurability}

Measurability and source characteristics as indicators of ADR data quality are interconnected and context-dependent. Therefore, we consider them together.

In official sources, such as instructions for medicines, different scales are used. The measured parameter can be the frequency of occurrence of a side effect, but it can be measured on different scales. For example, for Atorvastatin, one gradation is used - either the frequency of ADR occurrence is more or less than $2 \%$. For Nifedipine, a linguistic scale "often - rarely - very rarely" is used.

There is also scaling according to ADR severity. For example, for Aspirin Cardio the gradations used are "contraindicated" and "with caution". However, we did not find simultaneous scaling of ADRs in terms of frequency of occurrence and severity of consequences, as required by the recommendations of the WHO (see section 2.2).

The characterization of official sources of ADR information is the responsibility of the relevant authorities and is outside the scope of this article. However, our analysis shows that now it is no longer possible to ignore network sources of information about ADRs where end-users of medicines leave messages, whether they are doctors or patients.

Our study showed a decline in user activity on online forums related to ADRs. So, the most popular drug on the forums (Atorvastatin) had a little more than 30 reviews, which is critically small for a full analysis of text data. At the same time, there is a growing popularity of discussing issues related to ADR in social networks. For example, from the social network VKontakte it was possible to collect 1.97 times more reviews than from all medicine forums combined.

In addition, we noticed the peculiarity of the comments about medicines in the Russian-speaking audience compared with the English-speaking one: in the first case, opinions are more often expressed in polar ("perfectly - terrible"), in the second case (for example, on DailyStrength Internet-resource) a wider range of opinions is presented. This must be taken into account when forming a scale for assessing the reliability of information about ADRs. 


\subsection{Summary Characteristic of ADR Data Quality from Various Sources}

The analysis performed allows us to characterize the quality of ADR data available in Russia at a substantive level.

The actuality of the data is not fully respected; even in official sources, there are examples of late updates of data on specific medicines, leading to ADR information out of sync.

The relevance of ADR data in the realities of modern Russia cannot be provided only by official sources. As analysis shows, data on the use of drugs after clinical trials, i.e. in real practice, published by end consumers, are practically not involved in official sources. At the same time, extracting data on ADRs from network sources today is quite easily implemented in software: modern Russian-language NLP systems oriented to extracting ADRs provide fairly good accuracy (F-score 0,8-0,9), and new algorithms are constantly being offered to improve it.

Data integrity is significantly violated (see section 4.1). The examples we demonstrate raise the question of the degree of trust in publicly available data on side effects. It should be noted that the problem of the integrity of ADR data is acute not only for Russia (see, for example, [Soyer, Mavrogiorgou, Jeong, Oh]), but, as far as we know, it is not considered in the Russian scientific literature even at the level of problem statement.

The measurability of data in official sources is supported, but different scales are used, which makes it difficult to share data in digital processing of medical information.

As can be judged by the results of the analysis, the data on ADRs obtained from official sources weakly correlate with similar data from spontaneous sources, at the same time, the popularity of messages on ADRs in the VK network is quite high, and this source of information on ADRs can no longer be ignored. However, for its introduction into clinical practice, it is necessary to develop appropriate methodological support.

\section{WAYS TO IMPROVE ADR DATA QUALITY}

The analysis confirms the opinion [Lepakhin] that the system for collecting and recording ADR data in Russia needs to be improved, which requires significant resource investments and organizational changes. The following are measures that can be seen as steps towards improving the quality of ADR data from spontaneous sources.

(1) In the database on the interaction of medicinal substances, it is advisable to enter the field "The degree of reliability of the primary source". A fragment of such a base is shown in the figure 5 .

The drug_substance1 and drug_substance2 tables contain information about medicinal substances. Each drug substance may be contained in several drugs. The type field - a scale for marking the degree of ADR - and the credibility field - the reliability of information on the interaction of drugs, are added to the Connection table. It is a lightweight and scalable solution that can be easily integrated into different types of databases. At the same time, it is consistent with WHO recommendations.

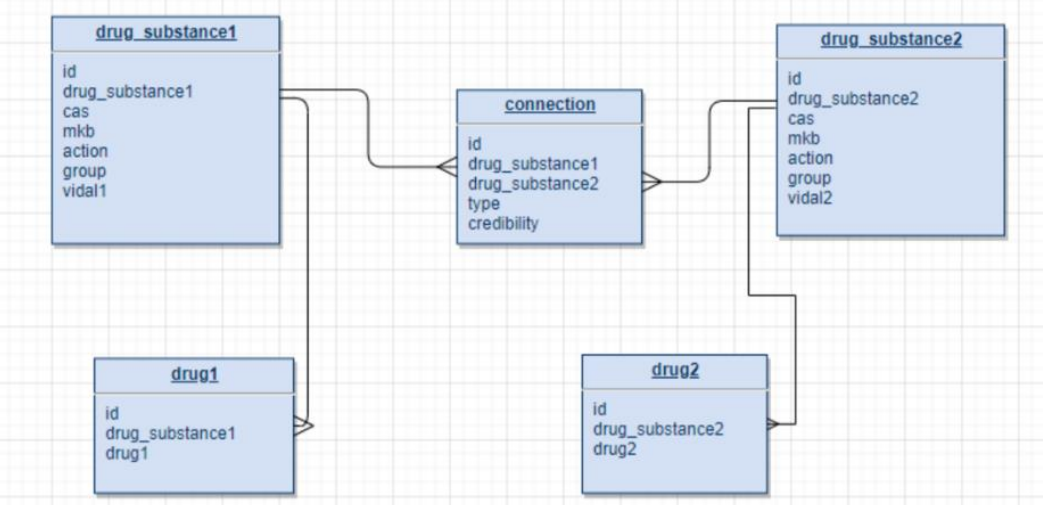

Figure 5. Database of drug interactions with fields describing the degree of interaction and the degree of reliability of primary information 
(2) For assessing the danger level of ADR manifestation, it is advisable to introduce a four-level scale similar to [Micromedex], and for the convenience of consumers, to label it with colors. An example of such a scale is shown in Figure 6 (note that this marking is used in Figure 3-4).

The use of such a scale simplifies the visualization of interaction matrices between drug substances using graphs such as Figure 3-5, allows the doctor and patient to integrally assess the situation and facilitates the decision-making on a specific drug. At the same time, to assess the degree of reliability of the primary source, it is sufficient to use a two-point scale, which corresponds to the WHO recommendations (see section 2.2) and at the same time is easily visualized on the drugs interaction graph.

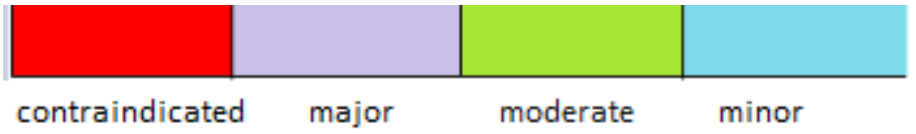

Figure 6. Proposed ADR grade scale

\section{CONCLUSION}

The results of quality analysis of data on ADRs for groups of drugs which are most frequently discussed by users of Internet resources were presented. The tasks set in the article have been fully carried out. Namely:

- A comparative analysis of data on ADR obtained from different sources available in Russia was conducted;

- The quality of ADR data available in Russia was evaluated. Problematic quality parameters of ADR data were identified - data integrity and measurability, as well as insufficient methodological support for retrieving and using the information from spontaneous data sources

- Ways are proposed to improve the quality of ADR data from spontaneous sources of information, which, on the one hand, will facilitate their use by end users, whether they are doctors or patients, and on the other hand, it will be easier to combine with ADR data from official sources.

\section{ACKNOWLEDGEMENT}

This work was financially supported by Russian Science Foundation, Grant 19-19-00696.

\section{REFERENCES}

[Antipov] Antipov E.A., Pokryshevskaya E.B. The effects of adverse drug reactions on patients' satisfaction: Evidence from publicly available data on Tamiflu (oseltamivir). International Journal of Medical Informatics 125 (2019) 30-36

[Baranov] Baranov A.A. et al. Technologies for Complex Intelligent Clinical Data Analysis. Annals of the Russian Academy of Medical Science. 2016; 71 (2). 160-171. doi: 10.15690/vramn663

Batini C. et al. Methodologies for data quality assessment and improvement. ACM Comput. Surv. 2009; 41, 3: Article 16, $52 \mathrm{p}$.

[Clinical] Clinical NLP in Languages Other Than English. https://amia2017.zerista.com/event/member/388973

[Doctors] Doctors rewiev about drugs. https://protabletky.ru/; Drug rating, http://topmeds.ru/, last accessed 2020/02/19.

Federal Service for Surveillance in Healthcare (Roszdravnadzor) Homepage, https://www.roszdravnadzor.ru/en, last accessed 2020/02/19.

[Foster] Foster JM, van der Molen T, de Jong van den Berg L (2007) Patient Reporting of Side Effects may provide an Important Source of Information in Clinical Practice. European Journal of Clinical Pharmacology 63: 979-980.

Good pharmacovigilance practices. European medicines agency. https://www.ema.europa.eu/en/human-regulatory/postauthorisation/pharmacovigilance/good-pharmacovigilance-practices, last accessed 2020/02/19.

[Jeong] Jeong Yun Park. Analysis of data on capecitabine-related adverse drug reactions from the Korean adverse event reporting system database. European Journal of Oncology Nursing 34 (2018) 55-60

[Khan] Khan L.M et al. Dilemmas of the causality assessment tools in the diagnosis of adverse drug reactions. Saudi Pharmaceutical Journal. Volume 24, Issue 4, July 2016, Pages 485-493 
[Lepakhin] Lepakhin V.K. et al. The landmarks of control and licensing system develioment for medicinal products un Russia (25 years since the establishment of the first state institution for expert evaluation of vedicinal products). Scientific Centre for Expert Evaluation of Medicinal Products Bulletin. 2016. №1. Pp. 3-10/

Lushnov [] Lushnov M. et al. Medical Knowledge Representation for Evaluation of Patient's State Using Complex Indicators // International Conference on Knowledge Engineering and the Semantic Web. - Springer International Publishing, 2016. - C. 344-359.

[Maheshwari] Maheshwari Paramasivam R. Sivabalakrishnan, N. Pooja. Evaluation of drug - drug interactions in urban area community pharmacies. Research Journal of Pharmaceutical, Biological and Chemical Sciences 7(4):1420-1424. January 2016

[Marante] Marante K.B. The Challenges of Adverse Drug Reaction Evaluation. J Pharmacovigil 2018, 6:3.

[Mavrogiorgou] Mavrogiorgou A. et al. Analyzing data and data sources towards a unified approach for ensuring end-toend data and data sources quality in healthcare 4.0. Computer Methods and Programs in Biomedicine 181 (2019) 104967

[MetaMap] MetaMap - A Tool for Recognizing UMLS Concepts in Text. https://metamap.nlm.nih.gov/, last accessed 2020/02/19.

[Micromedex] Micromedex Drug-Reax (2017). IBM Watson Health, Greenwood Village, Colorado, USA. Available at: https://www.micromedexsolutions.com/, last accessed 2020/02/19.

[Mosley] Mosley M., \& Brackett, M. (2010). The DAMA guide to the data management body of knowledge (DAMA-DMBOK guide), first edition. Bradley Beach, N.J.: Technics Publications.

[Na Hong] Na Hong et al. A computational framework for converting textual clinical diagnostic criteria into the quality data model. Journal of Biomedical Informatics 63 (2016) 11-21

[Oh] Oh I-S. et al. (2019) Differential completeness of spontaneous adverse event reports among hospitals/clinics, pharmacies, consumers, and pharmaceutical companies in South Korea. PLoS ONE 14(2): e0212336. https://doi.org/10.1371/journal. pone.0212336

[Prueksaritanont] Prueksaritanont T. et al. Drug-drug interaction studies: regulatory guidance and an industry perspective. AAPS J. 2013 Jul;15(3):629-45. doi: 10.1208/s12248-013-9470-x. Epub 2013 Mar 30.

[Romero] Romero F.P. et al. An approach to web-based Personal Health Records filtering using fuzzy prototypes and data quality criteria. Information Processing and Management 48 (2012) 451-466

[RMR] Russian Medicines Register. https://www.rlsnet.ru/rlsnetshop_show_details_82.htm, last accessed 2020/02/19.

[Soyer] Soyer J. et al. Identification of discrepanies between adverse drug reactions coded by medical records technicians and those reported by the pharmacovigilance team in pediatrics: An intervention to improve identification, reporting, and coding. Archives de Pediatrie 26 (2019) 400-406

[Talbot] Talbot J., Aronson J.K. (Eds.) Stephens' Detection and Evaluation of Adverse Drug Reactions: Principles and Practice, Sixth Edition (2011)

[Van den Berghe] Van den Berghe S., Van Gaeveren K. Data quality assessment and improvement: a Vrije Universiteit Brussel case study. Procedia Computer Science 106 (2017) 32 - 38

[Vatian] Vatian A. et al. (2018) Adaptation of Algorithms for Medical Information Retrieval for Working on Russian-Language Text Content. In: Sojka P., Horák A., Kopeček I., Pala K. (eds) Text, Speech, and Dialogue. TSD 2018. Lecture Notes in Computer Science, vol 11107. Springer, Cham

[Zhang] Zhang P. et al. Label Propagation Prediction of Drug-Drug Interactions Based on Clinical Side Effects. Nature. Scientific Reports 5, 12339 (2015). https://doi.org/10.1038/srep12339 